\title{
Tunable resonant optical microcavities by self-assembled templating
}

\author{
G. Vijaya Prakash, L. Besombes, T. Kelf, and Jeremy J. Baumberg \\ School of Physics \& Astronomy, University of Southampton, Southampton SO17 1BJ, UK
}

P. N. Bartlett and M. E. Abdelsalam

School of Chemistry, University of Southampton, Southampton SO17 1BJ, UK

Received January 15, 2004

\begin{abstract}
Micrometer-scale optical cavities are produced by a combination of template sphere self-assembly and electrochemical growth. Transmission measurements of the tunable microcavities show sharp resonant modes with $Q$ factors of $>300$ and 25 -fold local enhancement of light intensity. The presence of transverse optical modes confirms the lateral confinement of photons. Calculations show that submicrometer mode volumes are feasible. The small mode volumes of these microcavities promise to lead to a wide range of applications in microlasers, atom optics, quantum information, biophotonics, and single-molecule detection. () 2004 Optical Society of America

OCIS codes: $320.7090,140.4780,260.3910,140.3410$.
\end{abstract}

The operation of every type of laser, as well as of many varieties of atomic traps and optical sensors, depends on the formation of an optical cavity. The resonant optical modes inside this cavity determine the spatial field distribution and spectral performance of the device. The most common optical designs use spherical mirrors to form a confocal cavity that is insensitive to misalignment and confines the optical mode to the smallest lateral dimensions. ${ }^{1}$ There has been much interest in reducing the dimensions of such cavities: planar microcavities have been widely used to control spontaneous emission and to enhance the interaction of light with matter, as in quantum wells ${ }^{2}$ and quantum dots, ${ }^{3}$ but these structures confine photons in only one dimension. Confinement in lateral dimensions such as in photonic crystals ${ }^{4}$ and microcavity mesas ${ }^{5,6}$ can inhibit spontaneous emission altogether but involve complex and expensive fabrication strategies. Here we present a simpler approach that utilizes confocal microcavities. Whereas traditional lasers built from discrete components use macroscopic spherical mirrors, microcavity lasers do not. Preliminary theoretical work on the mode structure in parabolic dome ${ }^{7}$ and spherical ${ }^{8}$ cavities highlighted the promise of such zero-dimensional microcavities but confirmed that their fabrication is nontrivial. Similarly, glass and polymer microspheres show high $Q$ factors in whispering-gallery modes, but it is generally hard to control them and to couple light into and out of them. ${ }^{9}$ To construct cavities with the smallest possible mode volume requires small radius-of-curvature mirrors. Small mode volume vertical cavity surface-emitting semiconductor lasers require sensitive multistep lateral fabrication. ${ }^{5,10,11}$

Using our recently devised route to simple fabrication of such spherical micromirrors, we demonstrate here the formation of stable microcavities on size scales below $10 \mu \mathrm{m} .{ }^{12}$ To construct microcavities we combine spherical micromirrors of $\mathrm{Au}$ with radii
$R<10 \mu \mathrm{m}$ embedded in a thin film, with a planar-top $\mathrm{Au}$ mirror coated on a cleaved single-mode optical fiber. Both electron and optical microscopy (Figs. 1A and $1 \mathrm{~B})$ give an indication of the mirror's quality, discussed elsewhere. ${ }^{13}$ By growing the micromirrors on semitransparent substrates we take advantage of the thin Au coating at the base of the mirror to couple light into the cavity (Fig. 1C). The upper cavity mirrors are formed by evaporation of a $28-\mathrm{nm}$-thick $\mathrm{Au}$ film onto a perpendicularly cleaved stripped single-mode optical fiber that is mounted in an $X Y Z$ piezoelectric translation stage and aligned normally to the micromirror film. As the fiber approaches the film, optimizing the collected transmitted signal brings it into correct alignment with the micromirror, forming the microcavity. A white-light source is focused to a $10-\mu \mathrm{m}$ spot on the underside of the micromirror and imaged to allow us to backilluminate individual micromirrors. The detected light emerging from the fiber is the product of the cavity transmission
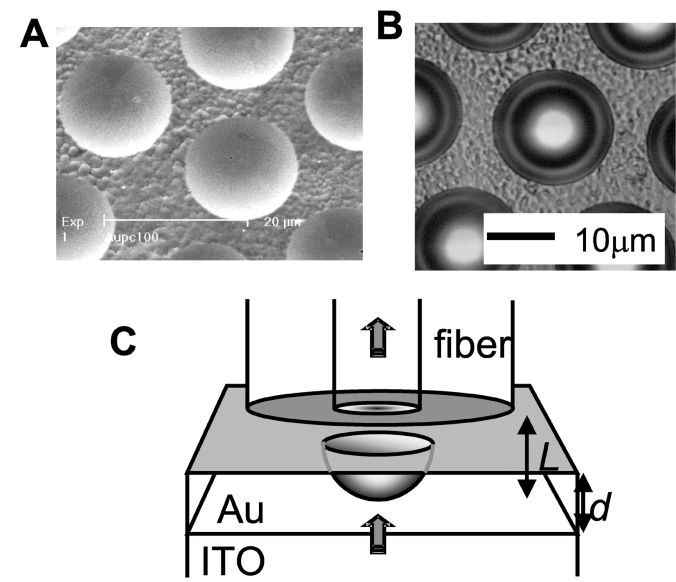

Fig. 1. Typical A, SEM and B, optical microscope images of spherical micromirrors $(R=10 \mu \mathrm{m})$. C, Microcavity design. ITO, indium tin oxide. 
and the coupling strength to the fundamental fiber mode. Using the fiber as the top mirror thus permits self-aligned detection geometry.

Spectral characterization of the optical transmission through the device allows us to compare the microcavities with simple models based on Gaussian beam optics. We also produce similar 28 -nm-thick plane $\mathrm{Au}$ reflectors that are combined with the same planar topfiber mirror to permit direct comparison with the plane-plane microcavity geometry (termed "planar" to distinguish it from the confocal plane-spherical mirror cavities). The general formula for the resonant frequency $\omega_{\mathrm{npl}}$ that produces zero net optical phase after one round trip is given by ${ }^{1}$

$\omega_{\mathrm{npl}}=\frac{c}{L}\left[\pi n-\theta_{\mathrm{Au}}+(2 p+l+1) \tan ^{-1}\left(\frac{L}{R-L}\right)^{1 / 2}\right]$,

where $n, p$, and $l$ are positive integers that describe the longitudinal, transverse radial, and azimuthal mode numbers of the resonance, respectively, the second term represents the phase shift on reflection from the metal film, and the third term is the Gouy phase shift that arises from the range of propagation angles contained in the Gaussian mode. From this paraxial approximation, the separation of the longitudinal modes yields the cavity length directly.

To prove that the spherical mirror is acting to localize the optical modes in the transverse direction we scan the core of the optical fiber laterally in the $X Y$ plane. Typical spectra for $R=10 \mu \mathrm{m}$ are shown in Figs. 2A and 2B and resolve a number of new optical modes (similar spectra are obtained for $R=5 \mu \mathrm{m}$ ). These modes always appear on the short-wavelength side of each original longitudinal mode and thus can be identified as transverse modes formed by lateral optical confinement of the spherical micromirror. Such modes are not observable in plane-plane microcavities. To further study the spatial dependence of the spectral transmission, we systematically collect spectra at different $Y$ positions and plot the resultant images. Scanning across the center of the microcavity (Fig. 2B; $X=0$ ) clearly shows the emergence of the transverse modes away from the cavity axis. As many as six transverse modes with similar linewidths make up each longitudinal mode. Scanning in the orthogonal direction gives similar results, indicating a near-cylindrical symmetry of the microresonator. Directly imaging the spatial mode distributions inside the microcavity thus allows us to show that the mode spectrum consists of discrete frequencies. Whereas an emitter inside a planar microcavity can always emit in some particular direction, an emitter in the confocal microcavity does not have this flexibility and can emit only in the appropriate field mode, $\omega_{\text {npl }}$.

In this cylindrically symmetric microresonator the field modes in the paraxial approximation are given by the Laguerre-Gauss functions ${ }^{1}$ plotted in Fig. $2 \mathrm{C}$ at the position of the top fiber mirror. This demonstrates how the microcavity dimensions here are nearly optimal for coupling cavity modes into the lowest guided modes in the fiber. We also note that the asymmetric modes possess a field null on axis that is useful for optical trapping of dark field species. ${ }^{14}$ The calculated lateral offsets that optimize collection of these various transverse modes are plotted as white crosses in Fig. 2B and show excellent agreement with the experimental data but for an effective $R=45 \mu \mathrm{m}$. In fact, as is visible from the micrographs of both Figs. $1 \mathrm{~A}$ and $1 \mathrm{~B}$ and in scanning force microscopy images (not shown), 10\% of the central area of the micromirrors is completely flat $(R=\infty)$, where the electrochemical growth appears to be screened under the template spheres (this effect scales with $R$ down to $R=100 \mathrm{~nm}$ ). Thus an effective cavity radius of curvature determined by both the curved and the flat sections is appropriate for the theory, in accord with the experimental results. However, the close match between experimental and theoretical finesse (see below) proves that this nonspherical mirror does not degrade the phase front of the lowest cavity mode and that mirror flatness is not a serious problem.

Both longitudinal and transverse mode separations decrease as the cavity length increases (Figs. 3A and 3B), as expected from Eq. (1). As was suggested previously, the predicted transverse mode separation accounts only for the data for an effective $R=45 \mu \mathrm{m}$. The difference between the planar and confocal microcavities becomes clearer when the transmission peak heights and linewidths are compared (Figs. 3C and $3 \mathrm{D})$. In the confocal geometry at cavity lengths $L<R=10 \mu \mathrm{m}$, strong optical field enhancement is observed and the finesse increases dramatically because of the reduced diffraction losses of the optical modes.
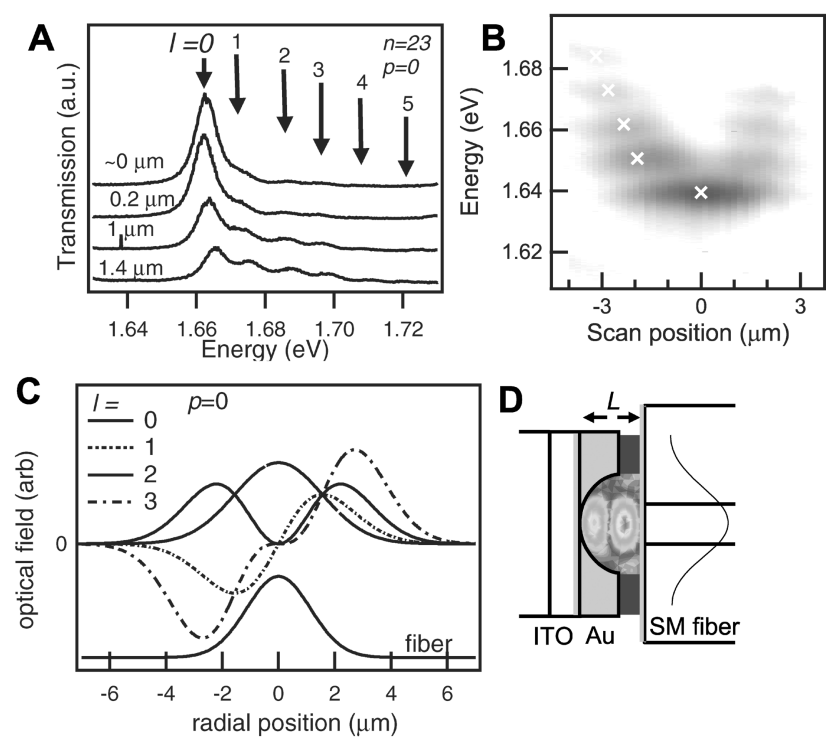

Fig. 2. A, Transmission spectra for confocal cavity length $L \sim 7.9 \mu \mathrm{m}, R=10 \mu \mathrm{m}$, with increasing lateral $X$ position. $B$, Transmission spectra of the same cavity (intensity on a log scale) versus lateral fiber $Y$ position at $X=0$. Crosses mark the predicted positions of the transverse modes collected by the fiber core. C, Optical field profiles for the fiber and the first four Laguerre-Gauss modes of the $R=10 \mu \mathrm{m}$ confocal cavity with $p=0$. D, Calculated field distribution in a spherical confocal microcavity on resonance; $R=880 \mathrm{~nm}, L=704 \mathrm{~nm}$. ITO, indium tin oxide; SM, single mode. 

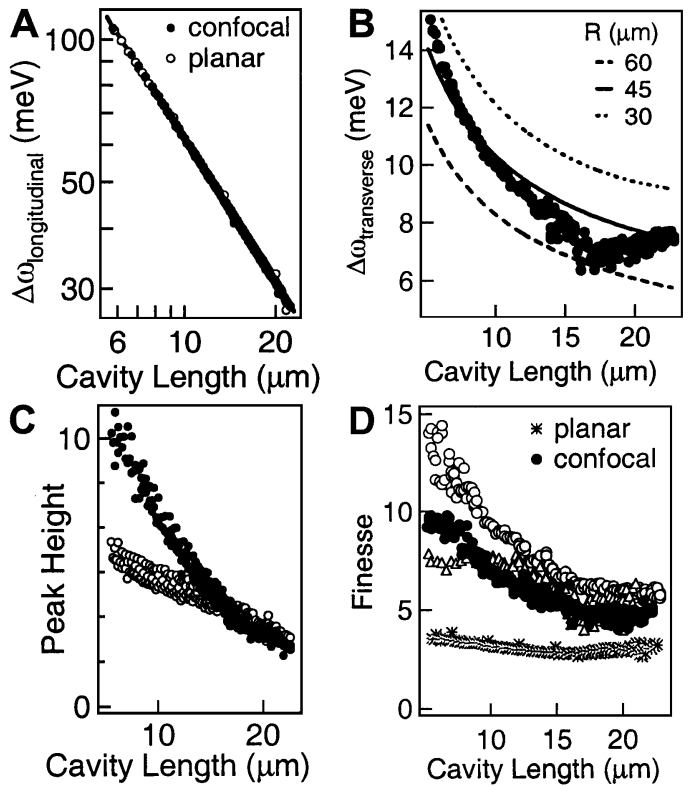

Fig. 3. A, Longitudinal cavity mode separations for planar and confocal cavities (the line shows the prediction from Gaussian optics). B, Transverse mode separation versus cavity length. C, Peak cavity transmission versus cavity length (, confocal cavities; $\bigcirc$, planar cavities). D, Cavity finesse versus cavity length for planar $(*)$ and confocal with one $(\bigcirc)$ and two $(\mathbf{O}, \triangle)$ transverse modes.

For the lowest transverse mode, a maximum finesse of $>15$ can be observed, giving $Q>300$. In contrast, the finesse of the planar cavity is $<5$, independently of cavity length. These results agree well with the theoretical finesse of $\pi \sqrt{r} /(1-r) \sim 14$, where $r=0.8$ is the product of the amplitude reflectivity coefficients of the two mirrors. The corresponding intensity concentrated within the cavity is $(1-r)^{-2} \sim 25$ times greater than that of the incident light at the resonant wavelength. This intensity enhancement occurs only at specific field antinodes within the cavity and is strongest for short cavity lengths. Higher-order transverse modes have a larger diameter on the cavity mirrors; hence they suffer extra loss and exhibit lower finesse, as shown in Fig. 3D.

Theoretical modeling to demonstrate the size scaling of these microcavities is carried out with a full solution of Maxwell's equations in these wavelength-scale geometries. Size scaling is not trivially possible in this system because of the much stronger influence of diffraction as the cavity length reaches the optical wavelength. The ultimate aim is to create the smallest optical cavities $(L \sim \lambda / 2)$ with the highest finesse. A typical electric field distribution is shown in Fig. 2D and corresponds to a mode volume below $1 \mu \mathrm{m}^{3}$; it shows that our templating scheme is suitable for submicrometer optical devices. For example, subpicosecond optical modulators that use resonant cavity enhancement of nonlinearities require short cavity lifetimes and hence short cavity lengths.

To summarize our key results, we have been able to fabricate near-spherical micromirror optical cavities with mode volumes below $5 \mu \mathrm{m}^{3}$ with $Q>300$, a finesse of as much as 30, and an intensity enhancement of $>25$. Both longitudinal and transverse optical modes are observed and can be simply wavelength tuned by piezoelectric transducer translation of the planar top mirror, which also extracts light from the cavity. In addition, this top fiber mirror can be replaced by high-reflectivity multilayers supporting luminescent nanoparticles, such as semiconductor quantum dots, for enhanced emission and microlasing. We note that metal mirror cavities with similar finesse have already been shown to be sufficient for attaining strong light-matter coupling in organic chromophores. ${ }^{15}$ Our preliminary measurements already suggest that these micromirror cavities are useful for optical-dipole force trapping, for enhanced collection of microphotoluminescence from the focal region, and for incorporation into experiments involving cold atom interactions on integrated chips.

We gratefully thank Ed Hinds and Tim Freegarde for helpful discussions of resonator design and Oliver Wright and Dave Hanna for critical comments. We acknowledge financial support from Engineering and Physical Sciences Research Council (UK) grants GR/N37261, GR/R54194, GR/S02662, and GR/ N18598. G. V. Prakash's e-mail address is prakash@ phys.soton.ac.uk.

\section{References}

1. A. E. Seigman, Lasers (University Science, Mill Valley, Calif., 1986).

2. H. M. Gibbs, F. Jahnke, M. Kira, and S. W. Koch, Rev. Mod. Phys. 71, 1591 (1999).

3. H. Saito, K. Nishi, I. Ogura, S. Sugou, and Y. Sugimoto, Appl. Phys. Lett. 69, 3140 (1996).

4. E. Yablonovitch, Phys. Rev. Lett. 58, 2059 (1987).

5. J. M. Gérard, D. Barrier, J. Y. Marzin, R. Kuszelewicz, L. Manin, E. Costard, V. Thierry-Mieg, and T. Rivera, Appl. Phys. Lett. 69, 449 (1996).

6. J. M. Gerard and B. Gayral, J. Lightwave Technol. 17, 2089 (1999).

7. J. U. Nockel, G. Bourdon, E. Le Ru, R. Adams, I. Robert, J. M. Moison, and I. Abram, Phys. Rev. E 62, 8677 (2000).

8. R. A. Abram, S. Brand, M. A. Kaliteevski, and V. V. Nikolaev, Phys. Status Solidi A 183, 183 (2001).

9. R. Jia, D.-S. Jiang, P.-H. Tan, and B.-Q. Sun, Appl. Phys. Lett. 79, 153 (2001).

10. J. L. Jewell, A. Scherer, S. L. McCall, Y. H. Lee, S. Walker, J. P. Harbison, and L. T. Florez, Electron. Lett. 25, 1123 (1989).

11. K. L. Lear and E. D. Jones, MRS Bull. 27, 497 (2002).

12. P. N. Bartlett, P. R. Birkin, and M. A. Ghanem, Chem. Commun. 2000, 1671.

13. S. Coyle, G. V. Prakash, J. J. Baumberg, M. Abdelsalam, and P. N. Bartlett, Appl. Phys. Lett. 83, 767 (2003).

14. V. G. Chavez, K. V. Sepulveda, S. C. Cerda, W. Sibbett, and K. Dholakial, Phys. Rev. A 66, 63402 (2002).

15. P. A. Hobson, W. L. Barnes, D. G. Lidzey, G. A. Gehring, D. M. Whittaker, M. S. Skolnick, and S. Walker, Appl. Phys. Lett. 81, 3519 (2002). 